\title{
La modernisation du droit de l'environnement : quelle(s) orientation(s) pour le fabricant de la norme?
}

The modernisation of environmental law: what is/are the direction(s) for legal rule-makers?

\section{Thierry Édouard}

\section{(2) OpenEdition Journals}

Édition électronique

URL : http://journals.openedition.org/add/338

DOI : $10.4000 /$ add.338

ISSN : 2606-1988

\section{Éditeur}

Presses universitaires de Rouen et du Havre

\section{Édition imprimée}

Date de publication : 1 juin 2016

Pagination : 139-164

ISBN : 979-10-240-0599-7

ISSN : 1955-0855

\section{Référence électronique}

Thierry Édouard, «La modernisation du droit de l'environnement : quelle(s) orientation(s) pour le fabricant de la norme? », Les Annales de droit [En ligne], 10 | 2016, mis en ligne le 08 janvier 2018, consulté le 10 décembre 2020. URL : http://journals.openedition.org/add/338 ; DOI : https://doi.org/ 10.4000/add.338 


\title{
La modernisation du droit de l'environnement : quelle(s) orientation(s) pour le fabricant de la norme?
}

\author{
Thierry ÉDOUARD
}

La question de la modernisation de l'action publique environnementale française préfigure-t-elle le droit de l'environnement à l'aube du $\mathrm{xxI}^{\mathrm{e}}$ siècle $^{1}$ ? On sait que la Charte de l'environnement, élevée au rang constitutionnel ${ }^{2}$, confère une valeur juridique haute à la norme environnementale française traduisant, mutatis mutandis, la conception philosophique d'une forme de déférence à l'endroit de la nature. Ce texte, désormais partie intégrante du bloc de constitutionnalité, semble attester, du point de vue de la «fondamentalisation» du droit de l'environnement, d'une relation symbiotique entre la nature et la perpétuation de l'espèce humaine. De prime abord, on est tenté de penser que l'homme, pris en tant que produit de la nature, serait en quelque sorte le nécessaire subordonné, de sorte que la communauté nationale reconnaîtrait la nature comme « une force sensible et intelligente ${ }^{3}$ » à l'instar de la vision

1. On se référera utilement à la contribution de la Société française pour le droit de l'environnement (ci-après «SFDE ») dans le cadre des états généraux de la modernisation du droit de l'environnement (ci-après «EGMDE»), 25 juin 2013 (61 p.), disponible en ligne à l'adresse suivante: http://wwwsfde.ustrasbg.fr/downloads/Autres/ Contribution $\mid \% 20$ de $\ \% 20 l a \mid \% 20 S F D E \backslash \% 20 a u x \backslash \% 20 E G M D E . p d f$ (consulté le 18 novembre 2015). La présente contribution s'inscrit dans la perspective de la COP 21 (21e conférence des parties à la convention-cadre des Nations unies sur les changements climatiques). L'enjeu de ces négociations multilatérales est d'adopter enfin, à l'échelle mondiale, un véritable accord contraignant sur la réduction des émissions de gaz à effet de serre.

2. L. const. $\mathrm{n}^{\mathrm{O}} 2005-205,1^{\mathrm{er}}$ mars 2005 relative à la Charte de l'environnement, $J O$ 2 mars 2005 , p. 3697 . Intégré au bloc de constitutionnalité, le droit à l'information environnementale fait partie, depuis lors, des libertés et droits fondamentaux. Pour une première analyse, voir Michel Prieur, "Vers un droit de l'environnement renouvelé », NCCC 2004, ${ }^{\circ} 15$.

3. Une telle conception de la nature va au-delà même des thèses élaborées par la plupart des auteurs se revendiquant du courant de la deep ecology fondé par Arne Naess 
qu'en ont les mystiques des sociétés préindustrielles ou des sociétés traditionnelles, affirmant que "la préservation de l'environnement doit être recherchée au même titre que les autres intérêts fondamentaux de la Nation». En fixant un objectif aussi élevé, le pouvoir normatif renoncerait alors à toute visée simplificatrice ${ }^{4}$ et simpliste ${ }^{5}$, dès lors qu'il s'agirait d'intervenir en matière de protection et de préservation de l'environnement. Pour les pouvoirs publics, le raisonnement conduirait à ne plus considérer qu'une seule face des choses, et à rendre moins complexe ce qui l'est par nature. Autrement dit, dans le cadre du droit de l'action publique globale de l'État et de ses démembrements, la modernisation recherchée de la norme coïnciderait avec une adaptation à la philosophie de son temps dont les soubassements sont issus de la théorie managériale, rythmée par l'obsession quantitative. À partir de l'analyse économique du droit, il est permis de postuler que l'acte de moderniser aujourd'hui, est dominé par ce que les chercheurs de l'École des hautes études en sciences sociales appellent "le statactivisme ${ }^{6}$ ", théorie consistant à passer du stade d'individu de masse à celui de l'individu social ${ }^{7}$.

Mais il y aurait tromperie à penser que cette pétition de principe du droit constitutionnel français de l'environnement implique que la nature ait des droits qui lui seraient propres et qui seraient distincts du droit

dans son ouvrage Écologie, communauté et style de vie, Paris, MF, 2009, 372 p. Pour une analyse critique de cette théorie, voir Fabrice Flipo, "La "deep ecology", un intégrisme menaçant ou un libéralisme non-moderne?», 30 août 2010, disponible en ligne à l'adresse suivante : http://www.sens-public.org/spip.php?article761।\&lang=fr (consulté le 18 novembre 2015).

4. "Rendre moins complexe, réduire à l'essentiel, débarrasser d'éléments non indispensables; faciliter une action» (définition disponible à l'adresse suivante: http: //www.cnrtl.fr/definition/simplifier (consulté le 18 novembre 2015).

5. Qui ne considère qu'une seule face des choses et qui, raisonnant en conséquence, simplifie outre mesure (http://www.cnrtl.fr/definition/simpliste, consulté le 18 novembre 2015).

6. Emmanuel Didier et Cyprien Tasset, "Pour un statactivisme. La quantification comme instrument d'ouverture du possible", Tracés, Revue de sciences humaines, $\mathrm{n}^{\mathrm{o}}$ 14, 2013, p. 123-140; Isabelle Bruno, Emmanuel Didier et Julien Prévieux (dir.), Statactivisme. Comment lutter avec des nombres, Paris, Zones, 2014, p. 272.

7. Dans son essai sur la démocratie, Gérard Mendel montre que ce n'est pas la démocratie, prise en tant que modèle sociétal, qui est en cause, mais bien l'une de ses expressions politiques, à savoir la démocratie représentative. L'auteur propose de repenser profondément la notion de pouvoir et de recourir à la démocratie participative pour permettre aux individus d'acquérir du pouvoir sur leurs actes et, mutatis mutandis, de "passer de l'individu de masse à l'individu social " (Gérard Mendel, Pourquoi la démocratie est en panne? Construire la démocratie participative, Paris, La Découverte, 2003. p. 8 et suiv.) 
objectif. D'abord parce que ce postulat n'est absolument pas véhiculé par la Charte de l'environnement, et il faut bien admettre que de telles préoccupations n'ont jamais animé le législateur constitutionnel. Ensuite, notre droit positif repose fondamentalement sur une conception cartésienne et anthropocentrée de la nature ${ }^{8}$, qui accorde le primat à la nature-objet ${ }^{9}$, laquelle tendrait plutôt à considérer la nature comme un instrument au service de l'homme, à l'inverse de la nature-sujet, notamment théorisée par Arne Naess ${ }^{10}$.

Ces premières considérations montrent l'importance de cultiver un esprit empreint de grande mesure vis-à-vis de la modernisation du droit de l'environnement. Car si l'acte de moderniser procède plutôt d'une action vertueuse, manifestant le souci pour son auteur d'adapter aux besoins, aux idées, aux progrès de l'époque en cours, l'idée de rénovation qui est introduite laisse plutôt subodorer l'ambition du mieux ou de l’utile $^{11}$ dont les critères paraissent répondre au concept, résolument moderne, d'intérêt général orchestré par le droit public ${ }^{12}$. Cette perspective conduit, dans un premier temps, à examiner la singularité de la participation du public dans la fabrication de la norme (1). Par la suite,

8. De plus amples développements seront abordés au 2.2 de la seconde partie de la contribution.

9. «Exiger la protection de l'environnement impose que l'homme se soumette à des obligations envers la nature, ce qui n'implique pas pour autant que la nature ait des droits» (Michel Prieur, "Vers un droit de l'environnement renouvelé », art. cité).

10. C'est d'ailleurs ce parti pris qui a été retenu par la commission Coppens, qui a profondément inspiré la loi constitutionnelle relative à la Charte de l'environnement. Sur ce point, voir Marie-Angèle Hermitte, «La nature, sujet de droit? ", Annales. Histoire, sciences sociales, 2011, $\mathrm{n}^{\mathrm{o}}$ 1, p. 173-212. Voir également Eduardo Gudynas, "Développement, droits de la Nature et Bien-Vivre: l'expérience équatorienne ", Mouvements, vol. 4, $\mathrm{n}^{\mathrm{0}}$ 68, 2011, p. 15-37: la référence explicite de la thèse de Gudynas est bien celle de l'écologie profonde (deep ecology) théorisée par Arne Naess, même s'il précise que «la formulation équatorienne s'est créée en bonne partie de manière indépendante, et l'apport des traditions et sensibilités des groupes indigènes, ainsi que le travail antérieur de nombreuses organisations environnementales n'y ont pas été négligeables ».

11. À ce propos, on notera par exemple que les ONG doivent avoir un but non lucratif d'utilité internationale pour qu'elles se voient appliquer les dispositions de la Convention européenne sur la reconnaissance de la personnalité juridique des organisations internationales non gouvernementales, signée le 24 avril 1986 (Conseil de l'Europe, traités européens, STE $\mathrm{n}^{\circ}{ }_{124}$ ). Voir également L'utilité publique aujourd'hui, étude adoptée par l'Assemblée générale du Conseil d'État le 25 novembre 1999, Paris, La Documentation française, 1999, p. 23.

12. On rappellera que la notion d'utilité publique est partie intégrante de l'intérêt général. 
il sera opportun d'envisager les contraintes philosophiques liées à cette évolution normative (2).

\section{La singularité de la modernisation du droit de l'environnement}

Il faut accepter l'idée que l'objet principal de la modernisation du droit de l'environnement est d'assurer l'effectivité du développement durable, étant rappelé qu'il s'agit d'obtenir un niveau élevé de protection de l'environnement dans le cadre du développement économique et social ${ }^{13}$. Cette finalité résulte de l'affermissement de la démocratie environnementale dans le temps (1.1) et sous-tend, parallèlement, des justifications démocratiques inhérentes à la modernisation du droit de l'environnement (1.2).

\subsection{L'affermissement de la démocratie environnementale dans le temps}

Avant tout, il importe d'observer que, même si le droit de l'environnement demeure relativement récent dans l'ordonnancement juridique français, il peine à se moderniser durablement. L'optimisation du processus de modernisation du droit de l'environnement (1.1.1) permet d'observer, parallèlement, l'affermissement du rôle du public dans le développement de ce «droit carrefour ${ }^{14} »(1.1 .2)$.

\subsubsection{L'optimisation du processus de modernisation du droit de l'environnement}

La finalité du processus envisagé est de déterminer les critères permettant de caractériser les éléments de modernisation du droit de l'environnement et de savoir s'ils s'inscrivent dans une perspective soutenable. $\mathrm{Si}$, jusque-là, les autorités nationales et européennes avaient la maitrise des questions environnementales, il est clair que, dans ce domaine, les politiques publiques sont désormais formulées au niveau régional

13. Ce concept a été défini en 1987 par la commission dite "Brudtland» comme étant «un développement qui répond aux besoins du présent sans compromettre la capacité des générations futures de répondre aux leurs ". Pour l'ONU, il s'agit d'un principe directeur fondamental (rés. 42/187, 11 déc. 1987).

14. Raphaël Romi, Droit de l'environnement, Paris, LGDJ, 8 éd., 2014, p. 15. 
et international ${ }^{15}$. Pour certains auteurs ${ }^{16}$, l'élaboration normative ne constitue plus le centre du politique et doit laisser la place à des soussystèmes spécialisés fondés sur des processus décisionnels plus souples et plus ouverts. Il est toujours possible de considérer, à cet effet, que l'idée de modernisation du droit renvoie aux notions d'efficacité et d'efficience, notions par ailleurs largement usitées dans le langage juridique. Ainsi, cette quête récurrente d' "optimal " n'est pas anodine et permet de traduire une certaine idée du " mieux » et de «l'utilité », sans perdre de vue que la finalité des initiatives a nécessairement vocation à satisfaire l'intérêt général. En partant du postulat que la modernisation du droit de l'environnement procède fondamentalement d'une action vertueuse manifestant le souci pour son auteur d'adapter aux besoins, aux idées, aux progrès de l'époque en cours, on observe comment le droit de l'environnement se distingue des autres branches juridiques, en ce sens qu'il insuffle une nouvelle forme de citoyenneté politique révélée par des concepts désormais inscrits dans le marbre constitutionnel ${ }^{17}$. Il est d'ailleurs révélateur d'observer comment le constituant de 1946 s'est fixé pour objectif de traduire dans le droit une certaine vision de la démocratie, où la participation de chacun procède non seulement d'un geste politique, le suffrage, mais également d'une philosophie de vie consistant à s'exprimer librement dans tous les domaines de l'action publique $^{18}$.

Ce faisant, on peut légitimement postuler que le processus de modernisation du droit de l'environnement ambitionne d'accorder le primat à l'intervention efficiente du public dans la définition et la mise en œuvre des politiques publiques environnementales. Cette forme de

15. Nations unies, Guide d'application de la convention d'Aarhus, p. 5, disponible en ligne à l'adresse suivante: http://www.unece.org/fileadmin/DAM/env/pp/ implementation $\backslash, \mid \% 2$ oguide/french/aigf.pdf (consulté le 18 novembre 2015).

16. Voir notamment: Patrick Le Gallès, «Les politiques locales et la recomposition de l'action publique », dans Denys de Béchillon, Jacques Caillosse et Didier Renard (dir.), L'analyse des politiques publiques aux prises avec le droit, Paris, LGDJ, 2000, p. 285 et suiv.; Pierre Muller, Les politiques publiques, Paris, PUF, 1998, p. 100 et suiv. ; Yves Mény et Jean-Claude Thoenig, Politiques publiques, Paris, PUF, 1989, p. 381 et suiv.

17. Par exemple, le principe de précaution, prévu à l'art. 5 de la Charte de l'environnement, au même titre que le principe de prévention, imposent d'agir en amont des risques de dégradation auquel l'environnement est exposé (voir Pascal Planchet, Droit de l'environnement, Paris, Dalloz, 2015. p. 46 et suiv.)

18. Tel est le sens du point 8 du préambule de la Constitution du 27 octobre 1946, qui prévoit le principe de participation des travailleurs à la détermination collective des conditions de travail et à la gestion des entreprises. 
gouvernance publique tendrait donc à améliorer la démocratie dite "administrative » pour cingler vers une conception plus large et plus complète du suffrage politique. Dans cette optique, la convention d'Aarhus ${ }^{19}$ s'inscrit dans la lignée de cette philosophie d'action publique dans la mesure où elle ambitionne la forme de participation la plus topique dans le processus décisionnel environnemental. Le principe d'information et de participation du public ${ }^{20}$, exprimé par ce que les praticiens appellent désormais la démocratie environnementale ${ }^{21}$, relève d'une démarche de légitimation de l'action publique. Parce qu'elle ambitionne d'approfondir l'action démocratique dans son ensemble, la démocratie environnementale s'avère une source incontournable de renouvellement du pacte social ${ }^{22}$.

Tout ceci ne vise à démontrer qu'une seule chose: l'émergence d'un nouveau paradigme destiné à répondre à l'idéal d'un bien-être mondial faisant partie de ce qu'on désigne désormais comme les «biens publics mondiaux ${ }^{23}$ ». Ainsi, la convention d'Aarhus ${ }^{24}$ montre comment la participation du public améliore non seulement la qualité mais

19. Convention relative à l'accès à l'information, à la justice en matière d'environnement et la participation du public au processus décisionnel adoptée à Aarhus le 25 juin 1998.

20. Introduits dans le bloc de constitutionnalité. Ils figurent tous les deux à l'article 7 de la Charte de l'environnement et constituent des «droits de citoyenneté ». Voir Gilles Dumont, La citoyenneté administrative, thèse de droit public, Université de Paris II-Panthéon-Assas, 2002, p. 213.

21. Conseil d'État, "La démocratie environnementale", intervention de Jean-Marc Sauvé, dans La démocratie environnementale aujourd'hui, Cycle 2011-2012, 17 novembre 2010, $4 \mathrm{p}$.

22. Ibid., p. 1.

23. Alain Barrau, Rapport d'information sur les relations entre l'Union européenne et les entités régionales, Assemblée nationale, rapport $\mathrm{n}^{\circ} 3211,28$ juin 2001, p. 75 et suiv.

24. La France a ratifié la convention d'Aarhus le 8 juillet 2002. Elle est entrée en vigueur le 6 octobre 2002 (L. $\mathrm{n}^{\mathrm{O}}$ 2002-285, 28 févr. 2002 autorisant l'approbation de la convention d'Aarhus et D. $\mathrm{n}^{\circ}$ 2002-1187, 12 sept. 2002 portant publication de la convention d'Aarhus). Le 28 février 2005, les parlementaires réunis en congrès ont adopté la charte de l'environnement. Désormais, le principe entre dans la Constitution française: "toute personne a le droit, dans les conditions et les limites fixées par la loi, d'accéder aux informations relatives à l'environnement détenues par les autorités publiques [...]»(art. 7). Les bénéficiaires des droits reconnus à l'article 7 de la charte sont toute personne physique ou morale, privée ou publique. Le droit d'accès à l'information ne porte que sur les informations détenues par les autorités publiques, conformément à la législation sur l'accès aux documents administratifs et à la convention d'Aarhus. L'article L. 110-1-4 du code de l'environnement pose comme principe général: «chacun a accès aux informations relatives à l'environnement, y compris celles relatives aux substances et activités dangereuses [...] » La participation du public aux décisions et politiques 
également l'efficacité des politiques publiques environnementales. Si ce texte ne propose aucune définition de la notion de "participation", il apporte des précisions importantes quant aux termes "public » et " concerné ", et permet d'y inclure les personnes physiques ou morales, à l'instar des associations, et les groupes.

C'est pourquoi la démocratie environnementale tend à évoluer à travers l'affermissement du rôle du public dans le processus de modernisation.

\subsubsection{L'affermissement du rôle du public dans le processus de modernisation}

D’une façon générale, on peut dire que le droit à la participation du «public concerné » ne s'appuie pas sur un intérêt suffisant ni même sur un intérêt juridique, celui de l'intérêt à agir. En réalité, il inclut tous les membres du public dont les droits garantis par la loi sont susceptibles d'être lésés par l'activité envisagée ${ }^{25}$. Par ce biais, le public est reconnu comme une source importante de préconisations, d'alternatives ou d'évaluations, piliers des politiques publiques, et permet d'optimiser le processus décisionnel. C'est probablement la même explication qui joue pour comprendre l'objectif assigné par le principe 1 de la déclaration de Stockholm (1972) qui reconnaît que «l'Homme a le devoir solennel de protéger et d'améliorer l'environnement ", et par le principe 10 de la déclaration de Rio sur l'environnement et le développement (1992) selon lequel «la meilleure façon de traiter les questions d'environnement est d'assurer la participation de tous les citoyens concernés au niveau qui convient ${ }^{26} »$. On ne manquera pas de souligner, également, que la Charte européenne sur l'environnement et la santé reconnât que la participation du public est l'élément central de la modernisation du droit de l'environnement puisque chaque citoyen a le droit de participer au processus de prise de décision ${ }^{27}$.

environnementales se définit par trois éléments: l'information, la participation et l'accès à la justice.

25. Marguerite Boutelet et Juliette Olivier-Leprince (dir.), La démocratie environnementale, Presses universitaires de Dijon, 2009, p. 20.

26. Sur ce point, voir notamment: Michel Delnoy, «Définition, notion de base et raison d'être et sources juridiques des procédures de participation du public » dans Benoît Jadot (dir.), La participation du public au processus de décision en matière d'environnement et d'urbanisme, Bruxelles, Bruylant, 2005, p. 7-28.

27. Charte européenne sur l'environnement et la santé adoptée à Francfort sous l'égide de l'OMS le 8 décembre 1989 (premier paragraphe). 
$\mathrm{Si}$, donc, la modernisation de l'action publique environnementale réclame la participation du public au processus décisionnel pour élaborer une forme de démocratie environnementale, cette évolution garantit les droits de participation et conduit à une transformation des comportements, des concepts et des procédures qui pourrait avoir des répercussions non négligeables sur les acteurs impliqués ou concernés par l'action publique environnementale. Ce qu'une partie de la doctrine désigne comme un modèle de "modernisation participative ${ }^{28}$ ", est caractérisé par une gestion collective des contraintes environnementales par extension de l'activité de l'État dans ce domaine précis. Mais en tant que telle, cette action politique ne peut mettre sous le boisseau le fait que les acteurs doivent non seulement faire l'apprentissage du processus décisionnel de façon à situer leur intercession le plus en amont possible des politiques publiques environnementales mais également maîtriser le processus normatif in fine. Autrement dit, parler de modernisation, quelle que soit la branche de droit retenue, c'est d'abord développer la transparence et la participation du public dans l'élaboration des décisions politico-administratives ${ }^{29}$. C'est également s'engager sur un terrain hasardeux, qui prétend se situer au centre des préoccupations liées à l'action publique environnementale, les débats qui ont lieu sur le sujet n'ayant pas permis de l'éclairer suffisamment ni de parvenir à une définition sûre à laquelle on pourrait se référer ${ }^{30}$.

Dans ces conditions, l'idée d'une justification démocratique de la modernisation du droit de l'environnement s'impose à partir du moment où l'on admet qu'il s'agit d'un droit prospectif. On peut donc soutenir que le processus de modernisation du droit de l'environnement résulte, d'abord, du degré d'implication du public dans la définition et la mise en œuvre des normes environnementales.

28. Marguerite Boutelet et Juliette Olivier-Leprince (dir.), La démocratie environnementale, op. cit., p. 27.

29. En effet, à des textes souvent anciens (comme les lois sur les travaux publics et les travaux d'intérêt général de 1807 et 1892) prévoyant des enquêtes publiques sous diverses formes, par exemple les enquêtes parcellaires, s'est ajouté l'article $1^{\text {er }}$ de l'ordonnance du 23 octobre 1958, repris aux articles R. 11.1 et suivants du code de l'expropriation, qui instaure l'enquête en vue de la déclaration d'utilité publique, mais aussi la loi du 12 juillet 1983, dite "Loi Bouchardeau», relative à la démocratisation des enquêtes publiques et à la protection de l'environnement, s'agissant des projets des personnes publiques ou privées susceptibles d'affecter l'environnement.

30. Sur ce point, voir : Conseil d'État, La démocratie environnementale, Paris, La Documentation française, 17 janvier 2013, 306 p. Voir aussi : Pascal Planchet, Droit de l'environnement, op. cit., p. 58 et suiv. 


\subsection{La justification démocratique de la modernisation du droit de l'environnement}

Le critère téléologique de la modernisation du droit de l'environnement, c'est-à-dire le but en vue duquel les normes sont adoptées, semble trouver les premiers éléments d'expression à travers la convention d'Aarhus (1.2.1). Par ailleurs, les facteurs temporels permettent d'évaluer les politiques publiques environnementales (1.2.2).

\subsubsection{Le critère téléologique de la modernisation du droit de l'environnement}

Il n'est pas sans intérêt de rappeler que cette source conventionnelle a été particulièrement déterminante pour la constitutionnalisation du droit de l'environnement, à tel point que son intégration dans le corpus juridique français n'aurait pas eu lieu si la France n'avait pas ratifié ce texte international ${ }^{31}$. Mais, ici, on se situe dans une autre perspective que celle de l'affermissement de la démocratie environnementale, dans la mesure où peu d'informations restent vraiment secrètes, à l'exception notable des politiques de défense nationale ou du nucléaire civil ${ }^{32}$. C'est pourquoi la modernisation de l'action publique environnementale révèle une conception fondamentalement téléologique du domaine envisagé. Autrement dit, l'examen de la législation environnementale montre, en effet, que c'est la résilience de la démocratie environnementale ${ }^{33}$ qui permet d'éclairer les décisions publiques en associant le public ${ }^{34}$ à tous les stades du processus décisionnel. Il n'est désormais pas contestable, ni même contesté, que le paradigme du contrat social n'est plus adapté aux fondements contemporains d'exercice du pouvoir, puisque l'interventionnisme d'État ne parvient pas à juguler durablement l'émergence d'un pouvoir environnemental local. Celui-ci, comme de nombreuses politiques publiques, est de plus en plus ouvert à la contractualisation de l'action publique ${ }^{35}$, à tel point qu'il parvient à concurrencer l'échelon

31. Michel Prieur, «La convention d'Aarhus sur l'information et la participation: une coutume internationale en marche", dans Carine David et Nadège Meyer (dir.), L'intégration de la coutume dans l'élaboration de la norme environnementale, Bruxelles, Bruylant, 2012, p. 479 et suiv.

32. Pierre Muller, Les politiques publiques, op. cit., p. 93 et suiv.

33. Conseil d'État, La démocratie environnementale, rapport 2012, op. cit., p. 56.

34. Dans sa conception la plus large.

35. Arnaud Cabanes, Essai sur la gouvernance publique, Paris, Gualino, 2004. p. 39 et suiv. 
central et européen. Cette forme si particulière de démocratie participative, que l'on retrouve également en droit de l'urbanisme ${ }^{36}$, conduit à perturber les règles de la démocratie élective en prétendant contrôler le pouvoir donné aux élus pendant leur mandat ${ }^{37}$.

Il s'ensuit que la raison d'être de la modernisation du droit de l'environnement est de contraindre les autorités légitimes par le biais de ces nouvelles formes de droit démocratique, dans la perspective d'élaborer un droit revendicatif, assurément militant ${ }^{38}$, à tel point que la notion de "décision ayant une incidence sur l'environnement", largement interprétée par le Conseil constitutionnel et le Conseil d'État ${ }^{39}$, justifie l'intervention du public dans l'élaboration des normes environnementales. De la même manière, les facteurs temporels peuvent trouver une application dans la quête d'optimisation des normes environnementales.

\subsubsection{Les facteurs temporels dans la modernisation du droit de l'environnement}

L'invocation d'une gestion temporelle des contraintes environnementales n'est pas anodine car elle permet d'évaluer les politiques publiques environnementales, au même titre que les autres référentiels. On y observe comment l'efficacité d'une politique environnementale dépend non seulement des moyens qui lui sont associés mais aussi des comportements qui sont mis en œuvre. C'est en cela que nous soutenons que seule une analyse diachronique ${ }^{40}$ permet de mettre en évidence la modernisation du droit de l'environnement. Mais il faut cependant prendre garde à ne pas caricaturer la réalité, dans la mesure où la justification de la modernisation constitue, du moins pour l'État, un moyen d'exercer une autre forme d'intervention, contribuant à maintenir une

36. Tel est le cas de l'enquête publique qui a longtemps été liée au droit de l'urbanisme avant de trouver des prolongements dans le domaine du droit de l'environnement.

37. Marguerite Boutelet et Juliette Olivier-Leprince (dir.), La démocratie environnementale, op. cit., postface.

38. Pascal Planchet, Droit de l'environnement, op. cit., p. 3 et suiv.

39. Notamment: Cons. const., déc. $n^{\circ}$ 2012-269 QPC, 27 juill. 2012 ; CE, req. $n^{\circ} 373671$, 23 juin 2014 (à propos des autorisations d'exploitation commerciale).

40. En linguistique, la diachronie est l'étude des phénomènes linguistiques considérés du point de vue de leur évolution dans le temps. En sciences politiques aussi : voir Madeleine Grawitz, Méthodes des sciences sociales, Paris, Dalloz, 1996 ou JeanLouis Loubet Del Bayle, «Les problèmes de l'observation », dans Jean-Louis Loubet Del Bayle (dir.), Introduction aux méthodes des sciences sociales, Toulouse, Privat, $2^{\mathrm{e}}$ éd., 1986, $234 \mathrm{p}$. 
démarche descendante (top down), à l'instar de la loi Barnier de $1995^{41}$ ou de la Charte de la concertation du 5 juillet $1996^{42}$ destinée à améliorer la participation du public à la conception des projets ${ }^{43}$. De même, à travers cette «modernisation participative ${ }^{44}$ » il ne s'agit pas, pour l'État, de partager véritablement le pouvoir de décision mais plutôt d'exercer autrement ses compétences dans des procédures qui lui permettent d'asseoir sa légitimité ${ }^{45}$.

Dès lors, si l'on considère que les sciences de la "nature » s'occupent de la réalité telle qu'elle est, tandis que les sciences "normatives » visent un devoir-être ${ }^{46}$, il y a, sous-jacents, des choix fondamentaux qui doivent être opérés par les acteurs publics (État, collectivités territoriales, société civile), ce qui viendrait conforter l'approche téléologique envisagée supra ${ }^{47}$. Cette problématique peut, dès lors, trouver un terrain fertile à la doctrine de $\mathrm{Kant}^{48}$, dans la mesure où elle porte sur des normes juridiques relatives "à l'état présent de la société, à ses besoins, à ses mœurs et aux lois positives elles-mêmes, qui la gouvernent ${ }^{49}$ ". Autrement dit, il s'agit de rendre compte des lois positives, les seules qui désormais existent juridiquement, comme le physicien des lois de la nature $\mathrm{e}^{50}$.

Mais un constat s'impose : bien que ces dernières années soient riches en contributions de toutes $\operatorname{sortes}^{51}$, il paraît essentiel d'examiner les

41. L. $\mathrm{n}^{\mathrm{o}}$ 95-101, 2 févr. 1995 relative au renforcement de la protection de l'environnement, JORF 3 février 1995, p. 1840.

42. Ministère de l'Aménagement du territoire et de l'Environnement, La charte de la concertation, 5 juillet 1996.

43. Ces règles n'ont cependant pas vocation à se substituer à la procédure de l'enquête publique, telle que prévue par la loi du 12 juillet 1983.

44. Marie-Hélène Bacqué, Henri Rey et Yves Sintomer (dir.), Gestion de proximité et démocratie participative. Une perspective comparative, Paris, La Découverte, 2004, p. 295.

45. C'est d'ailleurs le cas s'agissant notamment, de la Charte de la concertation du 5 juillet 1996.

46. Michel Villey, Philosophie du droit, Paris, Dalloz, 2008, p. 250 et suiv.

47. Henri Batiffol, «La philosophie du droit», Revue philosophique de Louvain, vol. 62, $\mathrm{n}^{0} 74,1964$, p. 376 et suiv. ou La philosophie du droit, Paris, PUF, 1960, 128 p.

48. Emmanuel Kant, Métaphysique des mœurs. "Première partie. Doctrine du droit", Paris, Bibliothèque des textes philosophiques, 2011, 420 p. Voir également Hans Kelsen, Théorie générale des normes, Paris, PUF, 1996. p. 79 et suiv.

49. Michel Villey, Leçons d'histoire de la philosophie du droit, Paris, Dalloz, 1957, p. 175.

50. Jean-Pascal Chazal, «Philosophie du droit et théorie du droit, ou l'illusion scientifique », Arch. phil. dr. 2001, p. 310.

51. On en veut pour preuve le rapport de recherche «Concertation, décision, environnement», Programme CDE APR 2008-2009 (rapport final: «La participation du 
contraintes philosophiques de la modernisation du droit de l'environnement pour tenter d'en percer les évolutions intrinsèques.

\section{Les contraintes philosophiques de la modernisation du droit de l'environnement}

En tenant compte des développements précédents, on observera que l'un des points à éclaircir porte sur la nature de ce qui devrait être modernisé dans le droit de l'environnement. Or, même s'il demeure relativement moderne dans l'ordonnancement juridique ${ }^{52}$, nous savons que ce domaine rencontre les plus vives difficultés à se moderniser ${ }^{53}$. Cet élément est d'autant plus édifiant que l'acte de moderniser n'a, de surcroît, aucune signification juridique, pas plus que la volonté, consubstantielle, de «simplifier» le droit de l'environnement. Mais en dépit de ces obstacles a priori dirimants, il est possible de postuler que le processus de modernisation renvoie aux concept de cohérence, d'efficacité et d'évaluation, pour aller vers ce qu'une partie de la doctrine appelle le «mieux-disant environnemental ${ }^{54} »$. Ce processus semble aujourd'hui dominé par ce que les chercheurs de l'École des hautes études en sciences sociales appellent «le statactivisme", notion comprise à la fois comme un slogan et un concept descriptif, utilisé pour qualifier les expériences visant à se réapproprier le pouvoir des statistiques ${ }^{55}$. À l'instar d'autres politiques publiques, le «statactivisme " permet d'inférer, de manière plus ou moins affirmée, une autre réalité que la réalité environnementale officielle, dans la mesure où les statistiques environnementales

public à l'élaboration des textes réglementaires nationaux en matière d'environnement en France et à l'étranger: exigences démocratique, nécessité juridique»), janvier 2013, t. I, p. 234 et suiv.

52. Pour s'en convaincre, on pourra utilement se référer aux travaux du professeur Gilles J. Martin, « Le droit de l'environnement, nouveau droit ou non droit? » dans Dominique Bourg (dir.), La nature en politique ou l'enjeu philosophique de l'écologie, Paris, L'Harmattan. 1993.

53. Contribution de la SFDE aux EGMDE, p. 3 et suiv. On rappellera utilement que la modernisation suppose également, pour les sociétés insulaires d'outre-mer, la reconnaissance de leur identité, à savoir le système coutumier et les rapports organisés des hommes avec leur environnement. Sur ce point, voir Régis Lafargue, «Le préjudice civilisationnel pour atteinte à l'environnement. Droit au cadre naturel et réalités socioculturelles : interdépendances et interdisciplinarité », Droit et société, vol. 1, $\mathrm{n}^{\mathrm{o}} 74$, 2010, p. 151-169.

54. Marie-Laure Lambert-Habib, "Décentralisation constitutionnel et environnement », Rev. jur. env. 2004, p. 17 et suiv.

55. Emmanuel Didier et Cyprien Tasset, "Pour un statactivisme...», art. cité, p. 123-140. 
deviennent de véritables arguments politiques. Cela vaut également pour le droit de l'environnement, où le concept de modernisation peut jouer un rôle émancipateur important pour les acteurs impliqués dans ce domaine. Mais les préalables procéduraux (2.1), associés aux contraintes morales (2.2), conduisent à tempérer la finalité de la modernisation du droit de l'environnement.

\subsection{Les préalables procéduraux dans l'acte de moderniser}

La volonté de concilier développement économique et protection de l'environnement, dans l'optique d'un développement durable, a conduit à l'émergence de nouvelles formes de gouvernance, en particulier de nouvelles formes d'implication du public. Mais celles-ci demeurent somme toute relatives (2.1.1), d'autant que la nature des informations ne permet pas, a priori, de rendre efficient le rôle du public dans l'élaboration de la norme environnementale (2.1.2).

\subsubsection{La relativité dans l'implication du public}

Il n'est pas contestable, ni même contesté, que la philosophie qui sous-tend le dispositif normatif environnemental cherche à renforcer le contrôle public de l'action administrative ${ }^{56}$. Sous l'effet des nouveaux droits de citoyenneté, on voit bien comment l'application du principe de participation à la procédure d'élaboration et de la mise en œuvre de projets particuliers (enquête publique) et d'actes réglementaires illustre cette philosophie. Ainsi, l'enquête publique, qui n’a jamais été remise en cause par les pouvoirs publics ${ }^{57}$, s'impose préalablement à tous les projets ayant une incidence sur l'environnement. Mais cette nouvelle forme de citoyenneté environnementale n'a pas pour objet, ni même pour effet, de se substituer à la citoyenneté politique. Elle n'ambitionne que de la compléter.

De fait, ce qui s'apparente à un système, relativement pusillanime, de codécision entre l'administration et le public en matière environnementale ne risque-t-il pas d'aboutir à une forme d'insécurité juridique et de compromettre la finalité d'intérêt général? Nous répondons par

56. Pour une analyse topique, voir Jean-Luc Pissaloux, «La démocratie participative dans le domaine environnemental», RF adm. publ. 2011, p. 123-137.

57. Selon le rapport d'Alain Richard, il n'est pas question de remettre en cause l'enquête publique, cette dernière ayant largement fait ses preuves (Alain Richard, «Rapport "Démocratie environnementale: débattre et décider" ", remis à $\mathrm{M}^{\mathrm{me}}$ Ségolène Royal, ministère de l'Écologie, du Développement durable et de l'Énergie, 3 juin 2015, 74 p.). 
la négative, dans la mesure où, d'un point de vue purement théorique, le développement de la démocratie environnementale est nécessairement lié à celui de la démocratie dans son ensemble. C'est pourquoi la modernisation du droit de l'environnement conduit nécessairement à s'interroger également sur la notion d'utilité publique ${ }^{58}$.

Mais la réfraction du système normatif révèle l'absence d'effectivité des principes de la citoyenneté environnementale, qui ne semble pas achevée, en dépit de progrès indéniablement obtenus ces dernières années. En réalité, le droit à l'information s'apparente davantage à un droit de diffusion de l'information qui, en dépit de l'obligation d'informer le public, même sans texte, en cas de risques connus pour la santé des individus, demeure souvent inopérant dans la pratique ${ }^{59}$. En d'autres termes, la notion d'utilité contenue dans le droit à la diffusion de l'information du public, qui pourrait traduire l'effectivité de la participation du public au processus décisionnel environnemental, relève davantage de l'incantation que de la réalité. De plus, le «statactivisme» environnemental affermit la culture du secret, souvent décriée par les praticiens ${ }^{60}$, et ne parvient pas à moderniser durablement la gouvernance écologique.

Ce constat semble confirmé par la nature des informations environnementales.

\subsubsection{La nature des informations environnementales}

S'il est établi que le public a vocation à se prononcer sur l'opportunité d'un projet, encore faut-il que les informations qui lui parviennent soient fiables et pertinentes. Or, il semble que seules les activités environnementales contentieuses, qu'elles soient administratives ou pénales ${ }^{61}$,

58. L'utilité publique aujourd'hui, étude adoptée par l'Assemblée générale du Conseil d'État, 25 novembre 1999, Paris, La Documentation Française, 1999.

59. Si l'on se réfère aux articles L. 124-1 à L. 124-8 du code de l'environnement (en particulier l'article L. 124-3, $2^{\circ}$, in fine), on s'aperçoit que l'effectivité relative du droit à la diffusion de l'information semble étrillée par des domaines tels que le secretdéfense, la sécurité nationale ou la politique extérieure de la France. Sur cet aspect, voir Anne Soulas, "Genèse et évolution du droit à l'information », dans Marguerite Boutelet et Juliette Olivier-Leprince (dir.), La démocratie environnementale, op. cit., p. 46.

60. Sur ce point voir « Rapport de la mission confiée à Corinne Lepage sur la gouvernance écologique. Ministère de l'Écologie, du Développement et de l'Aménagement durable», février 2008, p. 6.

61. Marguerite Boutelet, «L'accès à la justice, garantie ou succédané de la participation du public aux politiques et aux décisions environnementales ", dans Marguerite Boutelet et Juliette Olivier-Leprince (dir.), La démocratie environnementale, op. cit., p. 65. 
permettent de corriger, bon an mal an, les insuffisances de l'effectivité de la participation du public au processus décisionnel. Mais ce droit contentieux se heurte inévitablement à des limites inhérentes à la notion de démocratie environnementale, dans la mesure où l'effectivité relative du droit à l'information ${ }^{62}$ en matière environnementale vient en limiter l'intérêt. En effet, " alors que l'administration et les administrés sont censés se retrouver devant le juge administratif sur un relatif pied d'égalité, cette égalité des armes est totalement rompue par le fait que l'administration dispose en réalité du choix entre respecter les règles ou les modifier à sa guise, au besoin en actionnant discrètement le législateur ${ }^{63}$ ». Ce constat laisse un sentiment d'insatisfaction et tendrait à faire admettre qu'il existe une réelle césure entre l'accès à la justice en matière d'environnement et la participation du public au processus décisionnel, étant entendu que ce dernier s'apparente davantage à un droit de participer du public qu'à une véritable participation au processus décisionnel. On objectera que les recours juridictionnels, qui constituent l'un des éléments de modernisation du droit de l'environnement, contribuent, d'une certaine manière, à élaborer les décisions administratives et donc le droit ${ }^{64}$. Nonobstant, l'ambition de la modernisation du droit de l'environnement se heurte inévitablement aux limites juridiques y afférant. Cette forme de "régression ", tant décriée par une partie de la doctrine, est illustrée par l'article L 300-2 du code de l'urbanisme, dont les objectifs, comme les modalités, ne répondent pas aux exigences fixées par la convention d'Aarhus. En effet, l'article 6 de cette convention prévoit que le public doit être informé «au début du processus » ( $\$ 2$, al. 1) et que «la participation du public commence au début de la

62. Par exemple, l'objet de l'enquête publique est de permettre au public de se prononcer sur l'opportunité d'un projet. Mais les conclusions défavorables du commissaire enquêteur ne lient pas l'autorité compétente. (art. L. 123-1 du code de l'environnement).

63. Xavier Braud, «La portée de la participation du public: sanction et application des décisions de justice», dans Marguerite Boutelet et Juliette Olivier-Leprince (dir.), La démocratie environnementale, op. cit., p. 112.

64. «La contribution importante des recours formés par des associations de protection de la nature au droit de l'environnement conduit périodiquement certains représentants des maîtres d'ouvrage à souhaiter que l'accès à la justice leur soit restreint» (Marie-Hélène Aubert, "Rapport fait au nom de la commission des Affaires étrangères sur le projet de loi ( $\left.\mathrm{n}^{\circ} 3256\right)$, autorisant la ratification de la convention sur l'accès à l'information, la participation du public au processus décisionnel et l'accès à la justice en matière d'environnement », Assemblée nationale, document $\mathrm{n}^{\mathrm{O}} 3566,30$ janvier 2002, p. 18. 
procédure, c'est-à-dire lorsque toutes les options sont encore possibles et que le public peut exercer une réelle influence» $\left(\$ 44^{65}\right)$.

Il résulte de ces considérations que la modernisation du droit de l'environnement n'aura seulement la portée que les autorités publiques voudront bien lui accorder. On en veut pour preuve la loi Grenelle II qui, même si elle a sensiblement modifié les règles de participation du public, ne semble pas augurer l'effectivité de la modernisation. Le même constat est établi par le récent rapport Richard ${ }^{66}$, qui montre comment les processus participatifs ne semblent plus capables de penser l'intérêt général, lequel demeure l'apanage des collectivités publiques ${ }^{67}$.

Ces difficultés, qui paraissent insurmontables, sont confortées par les contraintes morales induites par la modernisation du droit de l'environnement.

\subsection{Les contraintes morales}

La question de savoir si la nature a des droits n'est pas récente et a donné lieu à de nombreuses contributions doctrinales, plus ou moins éclairantes à ce sujet ${ }^{68}$. Néanmoins, il est désormais bien établi, du moins en France, que la définition de l'environnement est principalement anthropocentriste ${ }^{69}$ en considérant le droit de l'environnement comme

65. C’est ainsi que la République slovaque a été condamnée par la CJUE en janvier 2013 pour avoir méconnu cette exigence: CJUE, 15 janvier 2013, Krizan, aff. C-416-10 (AJDA 2013, p. 338, chron. Michel Aubert, Emmanuel Broussy et Hervé Cassagnabère).

66. Commission spécialisée du Conseil national de la transition écologique sur la démocratisation du dialogue environnemental présidée par Alain Richard, Rapport "Démocratie environnementale: débattre et décider", 3 juin 2015, 74 p.

67. Le débat entre les deux conceptions, l'une utilitariste, l'autre volontariste, n'a guère perdu de son actualité et de sa pertinence. Il illustre, au fond, le clivage qui sépare deux visions de la démocratie: d'un côté, celle d'une démocratie de l'individu, qui tend à réduire l'espace public à la garantie de la coexistence entre les intérêts distincts, et parfois conflictuels, des diverses composantes de la société; de l'autre, une conception plus proche de la tradition républicaine française, qui fait appel à la capacité des individus à transcender leurs appartenances et leurs intérêts pour exercer la suprême liberté de former ensemble une société politique (Conseil d'État, «Réflexions sur l'intérêt général», Rapport public, 30 novembre 1998).

68. On pourra utilement se référer à Alain Létourneau, « Pour une éthique de l'environnement inspirée par le pragmatisme : l'exemple du développement durable», VertigO. La revue électronique en sciences de l'environnement, vol. 10, $\mathrm{n}^{\circ}$ 1, avril 2010, voir https://vertigo.revues.org/9541 (consulté le 2 novembre 2015).

69. Pour s'en convaincre, il suffit de se référer au rapport de la commission Coppens de préparation de la charte de l'environnement (http://www. ladocumentationfrancaise.fr/var/storage/rapports-publics/054000294.pdf (consulté le 5 novembre 2015), p. 9 et suiv. 
« tout le droit qui touche à l'air, à l'atmosphère, l'eau, le sol, les terres, les paysages, les sites naturels, les zones côtières ou marines et la diversité biologique, et au-delà, si le juge ou le législateur le veulent ${ }^{70}[\ldots]$ ». À cet égard, les travaux de François Ost ${ }^{71}$ montrent comment l'objet du droit de l'environnement est de parvenir à une nature-projet, relayant tant la nature-objet ${ }^{72}$ que la nature-sujet ${ }^{73}$, cette dernière accordant le primat à la sanctuarisation de l'environnement, au détriment du développement durable. Profondément inspirée de la pensée de $\mathrm{Kant}^{74}$ et de Jonas ${ }^{75}$, la théorie de la nature-projet permet, bon an mal an, de conforter la notion de développement durable et la "patrimonialisation de la nature ${ }^{76}$ " pour promouvoir l'interdépendance entre l'homme et la nature. Ce débat, revivifié par l'encyclique Loué sois-tu, rappelle que "rien de

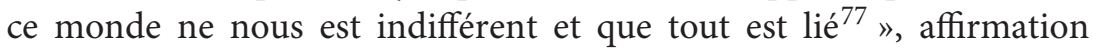
qui n'est, en soit, pas d'une grande originalité, mais qui postule que l'anthropocentrisme, même dévoyé, ne doit pas nécessairement faire place à un "bio-centrisme», parce que cela impliquerait d'introduire un nouveau déséquilibre qui, non seulement ne résoudrait pas les problèmes mais en ajouterait d'autres ${ }^{78}$. Pour autant, exiger la protection de l'environnement impose que l'homme se soumette à des obligations

70. Raphaël Romi, Droit de l'environnement, op. cit., p. 25 et suiv. Voir également Martine Rémond-Gouilloud, Le droit de détruire, essai sur le droit de l'environnement, Paris, PUF, 1989 , p. 25 et suiv.

71. François Ost, «La crise écologique : vers un nouveau paradigme? Contribution d'un juriste à la pensée du lien et la limite», dans Catherine et Raphaël Larrère (dir.), La crise environnementale, Paris, INRA, 1997, p. 39 et suiv.

72. La nature serait au service de l'homme (option passive).

73. La nature serait détentrice de prérogatives juridiques lui permettant de faire valoir ses propres droits face à l'homme (option active). En ce sens, Régis Lafargue préconise la reconnaissance de la personnalité juridique aux arbres, aux vallées et aux montagnes, qui agiraient en justice par le biais de représentants. Sur ce point, voir Régis Lafargue, «Le préjudice civilisationnel pour atteinte à l'environnement. Droit au cadre naturel et réalités socioculturelles : interdépendances et interdisciplinarité ", Droit et société, vol. 1, $\mathrm{n}^{\circ}$ 74, 2010, p. 151-169.

74. Emmanuel Kant, Métaphysique des mours, 1 re partie («Doctrine du droit»), Alexis Philonenko (trad.), Paris, Vrin, 1993, p. 106 et suiv. On pourra également utilement se référer à Michel Villey, «Kant dans l'histoire du droit », dans Leçons d'histoire de la philosophie du droit, Paris, Dalloz, $2^{\mathrm{e}}$ éd., 1962, p. 259 et suiv.

75. Sur ce point: Olivier Fuchs, "Le principe de responsabilité de Hans Jonas, contribution à l'étude de la médiation juridique des rapports de l'homme et de la nature», RRJ 2006, p. 1027 et suiv.

76. Raphaël Romi, Droit de l'environnement, op. cit., p. 27.

77. Lettre encyclique Laudato si du pape François sur la sauvegarde de la Maison commune, Rome, 24 mai 2015, p. 92 et suiv.

78. Lettre encyclique Laudato si, op. cit., p. 93. 
envers la nature, ce qui n'implique pas pour autant que la nature ait des droits $^{79}$. La commission Coppens, tout comme le préambule de la Charte de l'environnement, exprime ce nouveau paradigme politique lequel, bien que n'ayant pas de portée juridique contraignante, témoigne d'une véritable expression culturelle et d'une prise de conscience des enjeux à la fois planétaires et environnementaux des activités humaines.

Il n'en demeure pas moins que les justifications timorées de la protection juridique de la nature (2.2.1) se heurtent inéluctablement aux limites de la modernisation du droit de l'environnement (2.2.2).

\subsubsection{Les justifications timorées dans la protection de la nature}

Les constats précédemment opérés doivent toutefois être lus en tenant compte de la nécessaire protection de la nature, car l'exigence de protection de l'environnement impose que l'homme se soumette à des obligations envers celle-ci. Autrement dit, dans un contexte marqué par le changement climatique, par des dégradations majeures affectant les milieux naturels (érosion, pertes de biodiversité, pollutions...) ou encore par la raréfaction de ressources inégalement réparties dans l'espace (ressources fossiles, eau, terres arables...), le domaine de définition des choix et l'élaboration des décisions économiques ou politiques ne peuvent être considérés sans une réflexion sur les contours d'une nouvelle éthique environnementale pour affronter de nouveaux risques environnementaux et déterminer les réponses adéquates pour nous-mêmes et pour les générations futures ${ }^{80}$. Les récentes avancées consacrées au statut juridique de l'animal ${ }^{81}$ semblent illustrer les préoccupations actuelles des pouvoirs publics à l'égard des fruits de la nature et confirment que le corpus juridique national s'inscrit désormais dans la lignée du droit européen

79. Michel Prieur, «Vers un droit de l'environnement renouvelé», art. cité.

80. Sylvie Ferrari, Jean-Yves Goffi, Marie-Hélène Parizeau, Jean-Philippe Pierron et Éric Duchemin, «Éthique et environnement à l'aube du $21^{\mathrm{e}}$ siècle: la crise écologique implique-t-elle une nouvelle éthique environnementale?", VertigO - la revue électronique en sciences de l'environnement, vol. 10, $\mathrm{n}^{\circ}$ 1, avril 2010.

81. L. $\mathrm{n}^{\mathrm{o}} 2015-177,16$ fév. 2015 relative à la modernisation et à la simplification du droit et des procédures dans les domaines de la justice et des affaires intérieures (art. 2), JORF 17 février 2015, nº 0040, p. 2961. La loi a inséré un nouvel article dans le code civil - l'article 515-14 - rédigé en ces termes: «Les animaux sont des êtres vivants doués de sensibilité. Sous réserve des lois qui les protègent, les animaux sont soumis au régime des biens ». 
qui a considérablement modernisé le statut juridique des animaux, ou du moins renforcé les sanctions punissant leur maltraitance ${ }^{82}$.

De similaires observations peuvent être présentées à l'égard de l'objectif du droit de l'environnement, constituant d'ailleurs sa raison d'être, qui est la protection de l'environnement, opérant ainsi une véritable distinction avec les autres branches du droit. Désormais, la mobilisation juridico-politique s'applique à favoriser un «droit pour l'environnement ", qui ambitionne principalement la résolution des problèmes écologiques ${ }^{83}$, tout en plaçant l'homme au centre des préoccupations environnementales. Pour s'en convaincre, il suffit d'observer les principes de la déclaration générale adoptée lors du Sommet de la Terre organisé par l'ONU à Rio du 3 au 14 juin $1992^{84}$. Autrement dit, on ne peut pas parler de la nature sans parler des hommes, et du préjudice à l'environnement en éludant les "préjudices civilisationnels » ou «culturels ». En somme, aborder les choses qui nous entourent revient à interroger notre conception de l'homme et finalement du rapport à l'autre ${ }^{85}$.

Mais, d'une part, il paraît nécessaire d'affirmer que le droit de l'environnement ne saurait être le droit de la nature, puisqu'il s'agit fondamentalement de tendre vers un droit universel et humaniste. C'est en cela que le droit de l'environnement se distingue du droit de la nature, par ses objectifs et les moyens mis à sa disposition. On en veut pour preuve la notion de "biens publics mondiaux ${ }^{86}$ ", qui accorde le primat à l'intervention humaine en préservant, autant que faire se peut, le patrimoine commun des êtres humains ${ }^{87}$. D'autre part, la question de savoir si le "statactivisme", pris en tant qu'instrument d'expression politique susceptible d'optimiser la défense de la nature, mérite d'être posée, car le risque de biaiser les chiffres d'une politique

82. Sur ce point, voir notamment: Sénat, «Proposition de loi reconnaissant à l'animal le caractère d'être vivant et sensible dans le Code civil», 7 octobre 2013, «Exposé des motifs", p. 3 et suiv.

83. Pascal Planchet, Droit de l'environnement, op. cit., p. 4

84. Pour une approche synthétique, voir ibid., p. 3.

85. Régis Lafargue, «Le préjudice civilisationnel pour atteinte à l'environnement. Droit au cadre naturel et réalités socioculturelles...", art. cité.

86. Alain Barrau, «Rapport d'information sur les relations entre l'Union européenne et les entités régionales. Assemblée nationale", rapport $\mathrm{n}^{\circ}$ 3211, 28 juin 2001, p. 75 et suiv. Voir également Les biens communs environnementaux: quel(s) statut(s) juridique(s)?, colloque international organisé par le CRIDEAU-OMIJ (EA 3177) à la faculté de droit et des sciences économiques de l'université de Limoges les 13 et 14 octobre 2015, à paraître.

87. Jérôme Attard, «Le fondement solidariste du concept "environnement-patrimoine commun" ", RJE 2003, n², p. 161. 
publique environnementale n'est pas à écarter, dans la mesure où l'appropriation des règles de production statistiques permet, plus ou moins discrètement, de les adapter aux propres intérêts d'une catégorie ou d'un corps social, à l'instar du phénomène observé au sein de l'institution policière ${ }^{88}$.

Dès lors, il s'agit de savoir si la nature dispose de droits lui permettant d'être effectivement protégée renvoie à la question de la permanence de la nature, prise en tant qu'ensemble matériel et immatériel immuable ${ }^{89}$. Autrement dit, à partir du moment où les décideurs environnementaux reconnaissent l'avantage qu'il y a à utiliser les connaissances statistiques environnementales, ils élaborent précautionneusement les ressources dont ils ont besoin (études d'impact, consultation, enquête publique, débat public, etc.) et n'hésitent pas à recourir à des consultants dont la mission principale est d'emporter, le cas échéant, la conviction du public.

Il résulte de cela que l'objectif de conférer un ensemble de règles favorables au développement durable, illustré par la modernisation du droit de l'environnement, s'avère un exercice particulièrement ardu. Ces difficultés se heurtent inévitablement aux limites inhérentes à une telle entreprise.

\subsubsection{Les limites de la modernisation du droit de l'environnement}

Ce qui apparaît, à travers ces observations, c'est que le processus de modernisation du droit de l'environnement suppose la concordance de plusieurs éléments tant endogènes (le droit de l'environnement doit être compatible avec d'autres branches du droit, tel le droit de l'urbanisme

88. À tel point qu'il existerait une autre réalité que la version des pouvoirs publics. Le cas de la police nationale qui est soumise, depuis quelques années, à la «politique du chiffre » et ses thuriféraires la «culture du résultat» reposant fondamentalement sur la quantification de l'activité des agents. Les commissaires de precinct (équivalent à peu près à un arrondissement de Paris) avaient pour charge de quantifier leurs activités, de façon à rendre des comptes très régulièrement à la plus haute hiérarchie policière, ce qui devait leur permettre de prouver qu'ils avaient pris des initiatives et été particulièrement "proactifs». Dès que cet instrument fut mis en place, la criminalité enregistrée baissa de façon impressionnante. Pour atteindre de bons résultats quantifiés, le comportement individuel le plus facile peut consister à biaiser les chiffres. Sur ce point, voir Emmanuel Didier et Cyprien Tasset, Pour un statactivisme..., op. cit.

89. François Ost, La nature hors la loi. L'écologie à l'épreuve du droit, Paris, La Découverte, 2003, p. 29; Régis Lafargue, "Le préjudice civilisationnel pour atteinte à l'environnement. Droit au cadre naturel et réalités socioculturelles...", art. cité, p. 151-169. Pour une approche interdisciplinaire, voir Philippe Huneman, Les sciences de la nature et les sciences de l'homme, Paris, Ellipses, 2001, 64 p. 
ou le droit minier) qu'exogènes (il paraît impensable d'envisager la modernisation du droit sans apport de la philosophie du droit, cette dernière étant, en quelque sorte, la raison d'être). Mais, plus on avance dans l'analyse, plus on s'aperçoit que la Charte de l'environnement ne parvient pas à se combiner avec la convention d'Aarhus pour consacrer le nouveau droit de l'environnement, pour contraindre l'administration à publier les projets de décrets ou à prendre en compte les observations du public. Ces lacunes, tant décriées par la doctrine, ne permettent pas d'intégrer le processus de décision communautaire et international, d'autant que la convention d'Aarhus n'est que partiellement applicable en droit français ${ }^{90}$. Notre pays est donc bien éloigné des pratiques opérées au Canada et aux États-Unis, qui connaissent depuis plus de cinquante ans la fameuse procédure de la notice and comment, qui permet au public de participer efficacement à l'élaboration des décisions publiques $^{91}$.

Ainsi, bien que la France ait signé une trentaine de conventions internationales liées à l'environnement, elle ne les a pas ratifiées ${ }^{92}$. À supposer qu'elles le soient effectivement, les normes ne sont ni incorporées au droit français ${ }^{93}$, ni appliquées; la plupart du temps, elles sont ignorées par les administrations et peu utilisées par les praticiens, alors que leur utilisation contentieuse pourrait donner lieu à des résultats probants ${ }^{94}$. On ne peut donc pas parler de modernisation du droit de l'environnement, quelle que soit l'optique envisagée, sans déplorer l'absence d'évaluation des politiques environnementales, même si l'on a

90. En effet, les dispositions de la charte, qui renvoient au législateur le soin d'en préciser les modalités d'application, ne sont qu'indirectement invoquées devant le juge administratif. Sur ce point, voir le commentaire de Karine Foucher sous la décision du Conseil constitutionnel du 8 avril 2001 (AJDA 2011, p. 1158).

91. Michel Prieur, «Vers un droit de l'environnement renouvelé», art. cité, p. 11-14.

92. Par exemple, le protocole de Kiev sur les évaluations stratégiques environnementales. Le protocole sur l'évaluation stratégique environnementale, signé par 35 gouvernements et l'Union européenne en mai 2003 à Kiev, en Ukraine, devait entrer en vigueur le 11 juillet 2010.

93. C’est le cas, par exemple, de la Convention européenne du paysage signée à Florence le 20 octobre 2000. La convention vise à encourager les autorités publiques à adopter aux niveaux local, régional, national et international des politiques et mesures de protections, de gestion et d'aménagement des paysages européens. Elle concerne tous les paysages, extraordinaires et ordinaires qui déterminent la qualité du cadre de vie des populations. Le texte prévoit une approche souple des paysages dont les caractéristiques requièrent divers types de mesures allant de la stricte conservation à la véritable création, en passant par la protection, la gestion et l'amélioration.

94. Tel est le cas dans l'affaire dite « de l'étang de Berre» (CJUE, 15 juillet et 7 octobre 2004, aff. C-213-03). 
vu apparaître, ces vingt dernières années, des préoccupations tendant à l'évaluation des politiques publiques ${ }^{95}$ à l'instar de la politique de la ville ${ }^{96}$ ou d'autres secteurs considérés comme sensibles (la sécurité, l'action sociale, etc.). En effet, force est de constater qu'il n'existe pas d'indicateurs juridiques, ex ante et ex post, qui seraient utilisés systématiquement pour l'évaluation environnementale. Or, l'évaluation des politiques publiques environnementales s'avère un outil incontournable pour en maîtriser les effets. Dans cette perspective, il est toujours possible de privilégier l'efficience des politiques publiques environnementales ${ }^{97}$, au détriment de l'efficacité des mesures entreprises, cette dernière consistant à comparer les résultats obtenus par rapports aux objectifs recherchés. Dans ces conditions, le lien évaluation/légitimation de la modernisation du droit de l'environnement devrait s'entendre dans un contexte de certification environnementale, telle qu'observée dans le secteur privé. En somme, il n'y a pas de raison que l'idée de certification puisse s'étendre à l'action publique environnementale ${ }^{98}$.

On avancera, par ailleurs, que la judiciarisation du droit de l'environnement permet de le moderniser. Dans cette perspective, la notion de "préjudice écologique ", inspirée de l'article 2226 du code civil ${ }^{99}$, paraît être une référence exploitable dans la mesure où elle reposerait sur le principe de la réparation intégrale, observée par ailleurs en droit de la responsabilité ${ }^{100}$. Autrement dit, l'effectivité des droits de la nature

95. Ainsi, le décret $n^{\circ} 98-1408$ du 18 novembre 1998 relatif à l'évaluation des politiques publiques précise que l'évaluation "a pour objet d'apprécier, dans un cadre interministériel, l'efficacité de cette politique en comparant ses résultats aux objectifs assignés et aux moyens mis en œuvre».

96. Joseph Fontaine, «Évaluer les politiques. Dispositifs d'exception pour la ville et banalisation de l'action publique», dans Denys de Béchillon, Jacques Caillosse et Didier Renard (dir.), L'analyse des politiques publiques aux prises avec le droit, op. cit., p. 251 et suiv.

97. Qui consiste à comparer les résultats avec les moyens mis en œuvre.

98. Pour une analyse pertinente de la certification, voir Jean-Marie Pontier, « La certification, outil de la modernité normative », D., 1996, Chron, p. 355.

99. Article 2226 du code civil : «L'action en responsabilité née à raison d'un événement ayant entraîné un dommage corporel, engagée par la victime directe ou indirecte des préjudices qui en résultent, se prescrit par dix ans à compter de la date de la consolidation du dommage initial ou aggravé [al. 1]. Toutefois, en cas de préjudice causé par des tortures ou des actes de barbarie, ou par des violences ou des agressions sexuelles commises contre un mineur, l'action en responsabilité civile est prescrite par vingt ans [al. 2] ».

100. Tel est le cas s'agissant de la loi de 1985 sur les accidents de la circulation. On pourra également se référer à Marie-Pierre Camproux Duffrène, «Les modalités de réparation du dommage ", dans Chantal Cans (dir.), Responsabilité environnementale: prévention, imputation, réparation, Paris, Dalloz, 2009, p. 113 et suiv. 
suppose que soient quantifiées les conséquences indésirables, voire intolérables de mécanismes (centraux ou déconcentrés) des politiques publiques environnementales. Mais si le droit de la nature, garanti par l'existence de plusieurs institutions, telles la Commission d'accès aux documents administratifs, son effectivité est sujette à caution. En effet, la faiblesse des données, leur trop grande dissémination, associées à la «culture du secret ${ }^{101}$ ", ne parviennent pas à endiguer durablement ce phénomène. C'est pourquoi aussi il est possible d'affirmer que le développement de la démocratie environnementale présente certaines similitudes avec le concept constitutionnel de libre administration ${ }^{102}$, qui accorde le primat à la culture étatique dans le système politicoadministratif français ${ }^{103}$, pour opérer une limite optionnelle particulièrement dirimante pour les autres acteurs institutionnels.

Le processus protéiforme de modernisation du droit ne pouvait faire l'économie d'une étude théorique du droit. Assurément, le processus de modernisation du droit de l'environnement semble renforcer le caractère polysémique du terme "environnement ${ }^{104}$ ». Ainsi, au fur et à mesure que l'on réfléchit sur la modernisation du droit de l'environnement, que l'on veut effective, on découvre de nouvelles embûches conjoncturelles visant à affermir une certaine cohérence nationale que sous-tend l'intérêt général. Si le droit de l'environnement ambitionne de s'inscrire dans un mouvement plus vaste d'approfondissement de la pratique démocratique dans son ensemble, on pourrait considérer, au vu de l'évolution normative, que sa modernisation est au public ce que la liberté individuelle est aux personnes physiques: comme celle-ci et comme toutes les libertés, elle est loin d'être absolue et ses limites sont bien rappelées pas le législateur et le juge. Il s'agit davantage d'une liberté d'être que d'une liberté d'agir. Dans ces conditions, on comprend que la modernisation du droit de l'environnement est loin d'être achevée. Si une nouvelle philosophie de l'action publique environnementale devait intervenir, elle pourrait, notamment, inclure une réflexion sur le sens du droit dans l'environnement, pour dégager une nouvelle gouvernance démocratique. «L'analyse de système est le mode de pensée d'une société

101. Jean-Marc Sauvé, op. cit., p. 3.

102. Jacques-Henri Stahl, «Le principe de libre administration a-t-il une portée normative? » NCCC 2014, $\mathrm{n}^{\circ} 42, \mathrm{p} .32$.

103. Jean-Marie Pontier, «La notion de compétences régaliennes dans la problématique de la répartition des compétences entre les collectivités publiques ", RDP 2003, $\mathrm{n}^{\mathrm{o}} 1$, p. 193-237.

104. Pascal Planchet, Droit de l'environnement, op. cit., p. 1 et suiv. 
complexe ; le management public est le langage de l'État dans une société complexe $^{105}$.» Mais n'oublions pas aussi qu'il s'agit de tout changer pour que rien ne change ${ }^{106}$. S'il s'agit bien d'une forme de démocratie, au sens où elle peut être une source de renouvellement du pacte social, sa dimension participative demeure fondamentalement administrative ${ }^{107}$ et n'a pas pour objet, ni même pour effet, de l'élever au rang d'une démocratie politique $^{108}$. À dire vrai, la modernisation du droit de l'environnement peut être considérée beaucoup plus comme un agent de mise en œuvre de la volonté étatique que comme une option susceptible de gêner l'État ordonnateur. Dans ces conditions, la qualification d'un tel concept au sein duquel le triptyque modernité/utilité/effectivité paraît conforter une vue de l'esprit. Dès lors, l'invocation de la modernisation du droit de l'environnement ne doit pas tromper. Si cette évolution présente bien les apparences du droit, exprimée, notamment, par la gouvernance écologique ${ }^{109}$, elle s'apparente davantage à une technique managériale profondément inspirée du concept des politiques publiques ${ }^{110}$, dont l'objectif est de garantir in fine les intérêts de l'État dans un domaine prédéfini ${ }^{111}$. Ce constat laisserait plutôt accroire que la modernisation dont

105. Pierre Muller, Les politiques publiques, op. cit., p. 15.

106. Selon Giuseppe Tomasi di Lampedusa, « réformer n'est rien de plus que changer ce qui est nécessaire pour que rien ne change ». L'institutionnalisation de la participation du public au processus décisionnel se révèle, finalement, une démocratie chimérique dans la mesure où elle ne parvient pas à atteindre le niveau de la démocratie politique qui, du moins pour l'instant, est l'apanage des autorités publiques. Dans la configuration actuelle, non seulement les conditions d'une participation effective ne sont pas réunies mais en outre l'encadrement participatif ne permet pas de changer la qualité de la démocratie ni de la fonder sur une "éthique de la transparence dans les prises de décision" (Territoires, $\mathrm{n}^{\circ} 465$, février 2006, p. 74).

107. Pour Jean-Marc Sauvé, la démocratie environnementale s'inscrit dans le cadre d'une démocratie administrative, distincte de la démocratie économique et sociale. Voir Jean-Marc Février, "Les principes constitutionnels d'information et de participation ", Environnement, $\mathrm{n}^{\mathrm{o}}$ 4, avril 2005, comm. 35.

108. C'est en cela qu'il existe des similitudes avec le concept de libre administration des collectivités territoriales. Sur ce point, voir notamment Constantinos Bacoyannis, Le principe constitutionnel de libre administration des collectivités territoriales, $\mathrm{Pa}$ ris, Economica, 1993, p. 90 ; André Roux, «La libre administration des collectivités territoriales est-elle une liberté fondamentale? ", CCC 2002, p. 88 et suiv. Voir, plus récemment, Jacques-Henri Stahl, «Le principe de libre administration a-t-il une portée normative», CCC 2014, p. 31 et suiv.

109. Corinne Lepage, «Rapport sur la gouvernance écologique. Ministère de l'Écologie, du Développement et de l'Aménagement durable», op. cit., p. 6.

110. Pierre Muller, Les politiques publiques, op. cit., p. 23.

111. L'analyse de système est le mode de pensée d'une société complexe; le management public est le langage de l'État dans une société complexe (ibid., p. 15 et suiv.) 
il s'agit n'a pas, du moins pour l'heure, vocation à se substituer à l'intérêt général, dont la finalité demeure l'apanage des seules autorités publiques, à commencer par l'État ${ }^{112}$. Autrement dit, le droit de l'environnement, même modernisé, demeure une affaire d'État, central et déconcentré ${ }^{113}$, qui s'attelle à reléguer l'action des collectivités territoriales à une véritable portion congrue.

Docteur en droit

112. Ibid., p. 124-125.

113. Raphaël Romi n'hésite pas à utiliser le terme "d'omnicompétence " du préfet en matière de protection de la nature (Raphaël Romi, Droit de l'environnement, op. cit., p. 220 et suiv.) 